\title{
Two phosphodiesterases from Ustilago maydis share structural and biochemical properties with non-fungal phosphodiesterases
}

\author{
Charu Agarwal', David J. Schultz ${ }^{\dagger}$ and Michael H. Perlin ${ }^{* t}$ \\ Department of Biology, Program on Disease Evolution, University of Louisville, Louisville, KY, USA
}

Edited by:

Joshua D. Nosanchuk, Albert Einstein

College of Medicine, USA

Reviewed by:

Joshua D. Nosanchuk, Albert Einstein

College of Medicine, USA

Clayton Luiz Borges, Universidade

Federal De Goias, Brazil

*Correspondence:

Michael H. Perlin, Department of

Biology, University of Louisville, 139

Life Sciences Building, Louisville, KY

40208, USA.

e-mail:mhperl01@gwise.louisville.edu

${ }^{+}$Charu Agarwal, David J. Schultz, and

Michael H. Perlin have contributed

equally to this work.

\begin{abstract}
The dependence of Protein Kinase A (PKA) activity on cAMP levels is an important facet of the dimorphic switch between budding and filamentous growth as well as for pathogenicity in some fungi. To better understand these processes in the pathogenic fungus Ustilago maydis, we characterized the structure and biochemical functions of two phosphodiesterase (PDE) genes. Phosphodiesterases are enzymes involved in cAMP turnover and thus, contribute to the regulation of the CAMP-PKA signaling pathway. Two predicted homologs of PDEs were identified in the genome of $U$. maydis and hypothesized to be involved in cAMP turnover, thus regulating activity of the PKA catalytic subunit. Both umpde 1 and umpde2 genes contain domains associated with phosphodiesterase activity predicted by InterPro analysis. Biochemical characterization of recombinantly produced UmPde1 (U. maydis Phosphodiesterase I) and UmPde2 demonstrated that both enzymes have phosphodiesterase activity in vitro, yet neither was inhibited by the phosphodiesterase inhibitor IBMX. Moreover, UmPde1 is specific for cAMP, while UmPde2 has broader substrate specificity, utilizing cAMP and cGMP as substrates. In addition, UmPde2 was also found to have nucleotide phosphatase activity that was higher with GMP compared to AMP. These results demonstrate that UmPde1 is a bona fide phosphodiesterase, while UmPde 2 has more general activity as a cyclic nucleotide phosphodiesterase and/or GMP/AMP phosphatase. Thus, UmPde1 and UmPde2 likely have important roles in cell morphology and development and share some characteristics with a variety of non-fungal phosphodiesterases.
\end{abstract}

Keywords: cAMP phosphodiesterase, PKA, nucleotide phosphatase, U. maydis

\section{INTRODUCTION}

Morphological transformation from a yeast-like budding form to an infectious filamentous form associated with invasive growth within host tissue is an important requirement for many pathogenic fungi. The Basidiomycete fungal pathogen Ustilago maydis is an excellent model system to study this dimorphic transition and for the analysis of the molecular basis of plant pathogenicity (Bölker, 2001; Feldbrugge et al., 2004; Garcia-Pedrajas and Gold, 2004; Huai et al., 2004; Garcia-Pedrajas et al., 2008; Gouy et al., 2010). It can be cultured easily on artificial plates in laboratory settings and various molecular biology techniques can be applied to this system, such as, gene disruption, transformation etc. Also, its genome has been sequenced and is available publicly at http://mips.helmholtzmuenchen.de/genre/proj/ustilago (Kämper et al., 2006).

To penetrate and colonize plant tissue U. maydis requires dikaryotic, filamentous hyphae that result from fusion of haploid budding, yeast-like cells of opposite mating type (Bölker et al., 1992; Snetselaar et al., 1996). Infection results in the development of a tumor at the site of colonization and ultimately leads to production of diploid teliospores. These teliospores are then disseminated by air or water and germinate under favorable conditions, thus starting the life cycle anew (Banuett and Herskowitz, 1996).

Dimorphic transition in U. maydis is regulated by two conserved signaling cascades, namely, the MAPK (mitogen-activated protein kinase) and cAMP-PKA (cyclic AMP dependent-protein kinase A) signaling pathways (Lengeler et al., 2000; D'Souza and Heitman, 2001). Both pathways play important roles in sensing signals from the environment, e.g., nutrient availability, $\mathrm{pH}$, presence of lipids, and pheromones from opposite mating type, and in the transition between budding and filamentous growth forms (Klose et al., 2004; Martinez-Espinoza et al., 2004).

The U. maydis cAMP-PKA signaling pathway plays an important role in development and virulence (Gold et al., 1994; Lee et al., 2003). Components of cAMP-PKA signal transduction pathways are conserved among distinct fungal species as well as between distinct organisms, e.g., fungi to humans. Several components of the $U$. maydis PKA signaling pathway have been studied in detail (Figure 1). Mutations in the gene encoding the regulatory subunit, $u b c 1$, of PKA result in a constitutively active PKA and result in a multiple budding phenotype (Gold et al., 1997). In contrast, mutations in the genes encoding adenylate cyclase, (uac1) the alpha subunit of a heterotrimeric G protein (gpa3) or the catalytic subunit of PKA (adr1) result in a constitutive filamentous phenotype that is caused by low PKA activity (Barrett et al., 1993; Regenfelder et al., 1997; Durrenberger et al., 1998). Collectively, these studies clearly establish the importance of cAMP levels (and control of these levels) as an important aspect of PKA activity and subsequent control of growth forms.

Cyclic nucleotides are important secondary messenger molecules in cells and their cellular levels are regulated not only by controlling production, but also by cyclic nucleotide phosphodiesterases that 
deplete the levels of cyclic nucleotides. Phosphodiesterases (PDEs) hydrolyze the phosphodiester bond of cyclic nucleotides and convert them into monophosphates. There are 11 different families of phosphodiesterases that have been identified in humans according to sequence, regulation, biochemical properties and substrate specificity (Mehats et al., 2002). In contrast, fungi typically have two genes encoding cyclic phosphodiesterases (Ramanujam and Naqvi, 2010), with, for example, Saccharomyces cerevisiae and Cryptococcus neoformans each possessing a Class I and a Class II PDE. As one of the key components of cAMP-dependent signaling pathways, PDEs have been studied in detail in many fungal systems, including $S$. cerevisiae and Schizosaccharomyces pombe, in mammalian systems, and in human fungal pathogens such as, Candida albicans, C. neoformans (Sass et al., 1986; Nikawa et al., 1987; Hicks et al., 2005; Jung et al., 2005) as well as human parasites such as the trypanosomatids, Trypanosoma cruzi and T. brucei (Walter and Opperdoes, 1982; D’Angelo et al., 2004; Alonso et al., 2006, 2007).

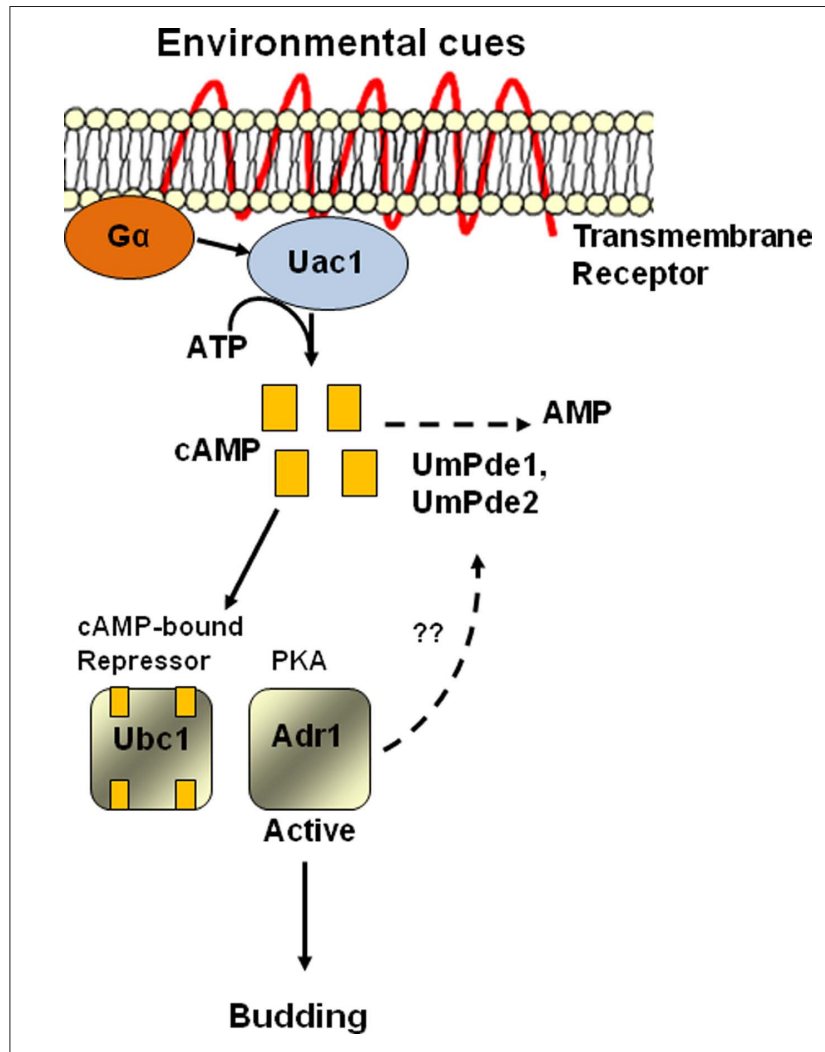

FIGURE 1 | Proposed model indicating roles of UmPde1 and UmPde2 in the cAMP signaling pathway. A morphogenic switch is regulated by the PKA pathway via several components. Adenylate cyclase (Uac1) is activated by Go-protein in response to environmental cues and converts ATP into cAMP. The CAMP binds with the regulatory subunit of PKA (Ubc1) causing it to dissociate from the catalytic PKA subunit. The active, derepressed catalytic subunit of PKA (Adr1) then targets downstream transcription factors and other proteins. UmPde1 and UmPde2 function to convert cAMP into AMP.This activity leads to continued repression of PKA activity due to Adr1 repression by Ubc1. Solid lines indicate known components of the pathway. The dashed line indicates a putative feedback regulation of UmPde1 and UmPde2 by phosphorylation through activated PKA. White squares of Ubc1 indicate unbound cAMP binding sites.
In addition to known roles in modulating/regulating cell morphology in fungi and metabolism in mammalian systems, PDEs have roles in stress response. For example, in S. cerevisiae, mutants deleted in PDEs fail to grow on rich media after 30 min of heat shock at $55^{\circ} \mathrm{C}$, thus indicating an important role in heat shock response (Sass et al., 1986). Another important step in the regulation of the level of secondary messenger molecules is compartmentalization of the activity of phosphodiesterases so that localized cAMP/cGMP signals can be generated to regulate different physiological responses. In T. cruzi, two membrane bound cAMP-specific phosphodiesterases have been identified; TcrPDEB2 is concentrated in the flagellum (D'Angelo et al., 2004), while TcrPDEC2 is possibly associated with vesicular membranes (Alonso et al., 2006), thus indicating the localized level of cAMP is important in this parasite (D'Angelo et al., 2004) as has also been shown for mammalian systems (Smith and Scott, 2006).

Levels of cAMP influence growth and development in a diverse array of organisms and can be altered either through increased production via adenylate cyclase or degradation via cAMP phosphodiesterase. Despite broad genetic studies of PDEs, there is a paucity of data on the biochemical nature of fungal PDEs, with S. cerevisiae and $C$. albicans being the primary fungal species for which biochemical characterization exists (Nikawa et al., 1987; Hoyer et al., 1994). Thus, as a first step toward a more complete understanding of the cAMP mediated cell morphology transition in $U$. maydis, we sought to analyze the sequence of PDEs in this organism and to better define the biochemical properties of the gene products. These $U$. maydis phosphodiesterases were found to have typical domains of phosphodiesterases based on InterPro analysis (Hunter et al., 2009). UmPde1, contains a PDEase-I domain, as well as having a predicted membrane-spanning domain and was found to be specific for cAMP using in vitro assays of recombinant protein; UmPde2, has a cAMP phosphodiesterase class II domain and, using in vitro assays of recombinant protein, was found to have a broader substrate specificity for both CAMP and cGMP. Along with phosphodiesterase activity, recombinant UmPde2 was also found to have nucleotide phosphatase activity wherein adenosine or guanosine 5 -monophosphates were converted to the corresponding nucleosides. Both of these PDEs are resistant to the known phosphodiesterase inhibitor, IBMX, a characteristic atypical of fungal PDEs examined so far, but also observed for some PDEs of Dictyostelium discoideum and for a subset of human and trypanosomatid PDEs.

\section{MATERIALS AND METHODS MEDIA AND STRAINS USED}

LB media (Yeast extract 0.5\%, Bacto-tryptone $1 \%$ and $\mathrm{NaCl} 1 \%$ ) was used for growth of bacteria. Escherichia coli strains, DH5 $\alpha$ (Bethesda Research Laboratories) and TOP10 (Invitrogen, USA), were used for all cloning and plasmid amplification. E. coli strains Rosetta2 (DE3) (Novagen, La Jolla, CA, USA) and BL21 (DE3) pLysS (Novagen) were used for expression analysis. Plasmid, pET30b, was purchased from Novagen (La Jolla, CA, USA). Nucleotides and IBMX were purchased from Sigma (St. Louis, MO, USA).

\section{ISOLATION AND EXPRESSION OF GENES}

Full-length ORFs of the genes were amplified by PCR. The pde1 gene was amplified using primers 02531 overexpA (5'-ATCGATC ACATATGAGCTCTGTCGTCGA-3') and 02531 overexpB (5'-CGT 
GCTGAATTCTTATAAACCGTCTCGAAGG-3') and the $p d e 2$ gene fragment was amplified using primers 10895 overexpA ( $5^{\prime}$-AGAGTA TCGCATATGTTCAATGGCAAGATCTGG-3') and 10895overexpB (5'-CGTGCTGAATTCTCAATACGCATGCCCTTTTCTG-3'). The $\mathrm{A}$ and B primers were designed, respectively, to contain NdeI and EcoRI restriction sites, indicated by italics Phusion DNA polymerase (Finnzymes, Lafayette, CO, USA) was used to reduce introduction of errors during PCR. Fragments were then cloned into TOPO TA vector (Invitrogen). The construct was then digested with NdeI and $E c o R I$ and the gene fragments were then cloned into pET30b vector, digested with the same enzymes, to obtain UmPde1-pET30b and UmPde2-pET30b constructs. The construct for expressing a variant of UmPde1 deleted for the membrane-spanning domain, AA87UmPde1-pET30b, was obtained in the same manner except the primers 02531-AA87OEPE (5'-ATCGATCACATATGCACAC CGTCCGACCGTG- $3^{\prime}$ ) (see Figure 2A, *, for location relative to predicted transmembrane domain) and 02531 overexpB were used. Restriction analysis was performed to check for the correct insert orientation and the constructs were then sequenced to verify the gene sequence.

The UmPde1-pET30b, UmPde2-pET30b and AA87UmPde1pET30b constructs and the empty pET30b vector were transformed into the BL21 (DE3) pLysS and the Rosetta2 (DE3) E. coli strains.

\section{IN SILICO ANALYSES AND PHYLOGENETIC INFERENCE}

InterPro searches were conducted on the MIPS U. maydis Database $\left(M_{U M D B}\right)$ to identify possible ORFs containing phosphodiesterase domains. Conserved domains were identified using the NCBI (National Center for Biotechnology Information) Conserved Domain Search Program ${ }^{2}$. Regions of hydrophobicity indicating possible membrane-spanning domains were screened using Protein Hydrophobicity Plot tool in the Molecular Toolkit ${ }^{3}$ and TargetP 1.1 (Emanuelsson et al., 2000) was used to search for presence of signal peptides indicative of targeting to sub-cellular organelles. Amino acid sequence alignments were performed with ClustalW (Thompson et al., 1994). The unrooted Phyl-ML trees were generated by the seaview4 package of phylogenetic software (Gouy et al., 2010), employing the WAG model for ML analysis. Support for major branches was calculated by phylogenetic analysis in PAUP4.0b10 using a Full Heuristic search with Bootstrap analysis of 1000 replicates.

\section{PRODUCTION OF RECOMBINANT PDE PROTEINS}

Overnight culture (4 $\mathrm{ml} \mathrm{LB}$ supplemented with appropriate antibiotics) was used as inoculum $(0.2 \%)$ for larger cultures ( $100 \mathrm{ml} \mathrm{LB}$ supplemented with appropriate antibiotics). Protein expression was induced by addition of IPTG to $0.75 \mathrm{mM}$ in larger cultures when they reached an absorbance at $600 \mathrm{~nm}\left(\mathrm{~A}_{600}\right)=0.4-0.6$.

To determine optimal protein expression, time course studies were used to assess production of recombinant protein in crude cell extracts. Aliquots $(2 \mathrm{ml})$ were taken at $0,2,4,8$, and $16 \mathrm{~h}$ after induction. For crude cell extracts, $1 \mathrm{ml}$ of culture was centrifuged at $10,000 \times g$ for $1 \mathrm{~min}$ and the supernatant was removed. Cell pellets

${ }^{1}$ http://mips.helmholtz-muenchen.de/genre/proj/ustilago

${ }^{2}$ http://www.ncbi.nlm.nih.gov/structure/cdd/wrpsb.cgi

${ }^{3}$ http://www.vivo.colostate.edu/molkit/hydropathy/index.html were resuspended in $1 \mathrm{ml}$ lysis buffer and sonicated (see below). For large-scale recombinant protein production, after harvesting cells from induced $100 \mathrm{ml}$ cultures, the sample was resuspended in $7 \mathrm{ml}$ lysis buffer $(50 \mathrm{mM}$ Tris- $\mathrm{HCl}, 5 \%$ Glycerol and $50 \mathrm{mM}$ $\mathrm{NaCl}, 7.5 \mathrm{kU} / \mathrm{ml}$ Lysozyme, $0.1 \mathrm{mM}$ PMSF and $25 \mathrm{U} / \mathrm{ml}$ DNase, $\mathrm{pH}$ 7-8) per gram of wet weight of pellet and was then sonicated on ice (Ultrasonic Homogenizer - Cole Parmer Instrument Co.) for at least five times for $30 \mathrm{~s}$ with an interval of $1 \mathrm{~min}$. The sonicated cell extract (crude enzyme extract) was then used for all further enzymatic analysis.

Protein concentrations were measured by Coomassie Plus Protein Assay (Pierce, Rockford, IL, USA). Protein was normalized in all the samples and then $10 \mu \mathrm{g}$ of protein was loaded onto a 4-15\% gradient Tris-HCl SDS-PAGE gel (Biorad, Hercules, CA, USA) to check for the expression of the protein of interest.

Gels were stained with Coomassie brilliant blue R250 in 40\% methanol, $40 \%$ water, and $10 \%$ acetic acid overnight. For de-staining $7.5 \%$ acetic acid was used and microwave was used to expedite the process.

\section{PHOSPHODIESTERASE ASSAY}

Phosphodiesterase assays were performed using crude protein extracts. The assay was modified from the protocol used by Abbott and Thompson (2005). Briefly, $0.1 \mathrm{mg}$ crude protein extract (described above) was supplied with substrate (1 mM cAMP, cGMP, AMP, ADP, ATP, GMP, adenosine, or guanosine) in $0.1 \mathrm{ml} 9 \mathrm{mM}$ HEPES buffer (Abbott and Thompson, 2005). Reactions were initiated by addition of protein and were incubated at $25^{\circ} \mathrm{C}$ for $1 \mathrm{~h}$ without shaking. Reactions were terminated by heating to $100^{\circ} \mathrm{C}$ for $3 \mathrm{~min}$. The samples were then stored at $20^{\circ} \mathrm{C}$ until analyzed by reversed phase HPLC.

\section{ANALYSIS BY RP-HPLC}

For analysis of assay substrates and products, Shimadzu LC-10 ADVP Liquid Chromatograph was used. Shimadzu's SPD-10ADVP UV-VIS detector was used for detection of peaks at $254 \mathrm{~nm}$. Prior to sample injection, the column (Shimadzu, premier RP-C18, $25 \mathrm{~cm} \times 4.6 \mathrm{~mm}, 120 \AA$ pore diameter, $5 \mu \mathrm{m}$ particle diameter) was equilibrated with $50 \mathrm{mM}$ ammonium di-hydrogen phosphate buffer with $10 \%$ methanol at a flow rate of $0.75 \mathrm{ml} / \mathrm{min}$. Samples were centrifuged to pellet the cell debris. Aliquots of $20 \mu \mathrm{l}$ from the supernatant were injected onto RP-HPLC column. Samples were injected manually by syringe with a Rheodyne injector, $7725 \mathrm{i}$, with $20 \mu \mathrm{l}$ SS sample loop. Isocratic conditions were used during the separation of nucleotides. Specific activity of crude protein extracts was calculated as nmol product formed $/ \mathrm{min} / \mathrm{mg}$ of crude protein. Activity of control samples (vector with no PDE insert) was subtracted in each case as background activity. The assays were performed in three independent replicates for each enzyme preparation and standard deviation was calculated.

\section{RESULTS}

\section{STRUCTURAL ANALYSIS OF PDES}

Currently, there are no annotations of ORFs as PDEs in MUMDB ${ }^{1}$. Possible U. maydis phosphodiesterases were identified by the InterPro domain search available on this website. Six putative ORFs were identified: um02531 (IPR002591, Type I phosphodiesterase/nucleotide 


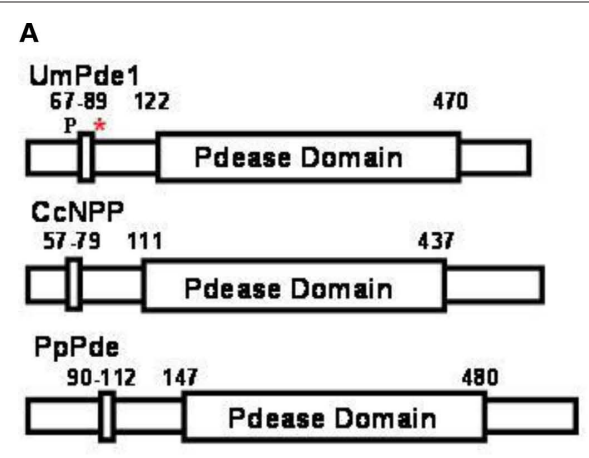

AcPde1

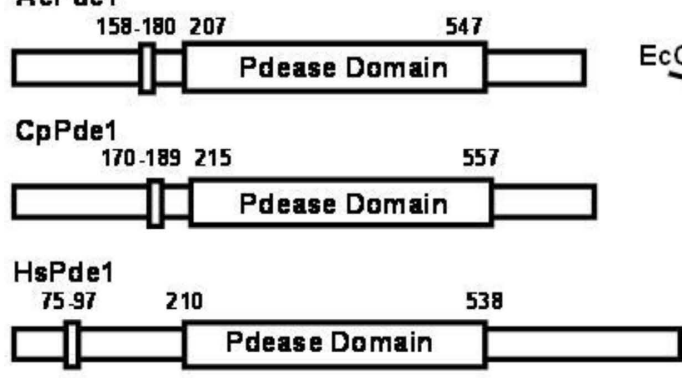

B
C

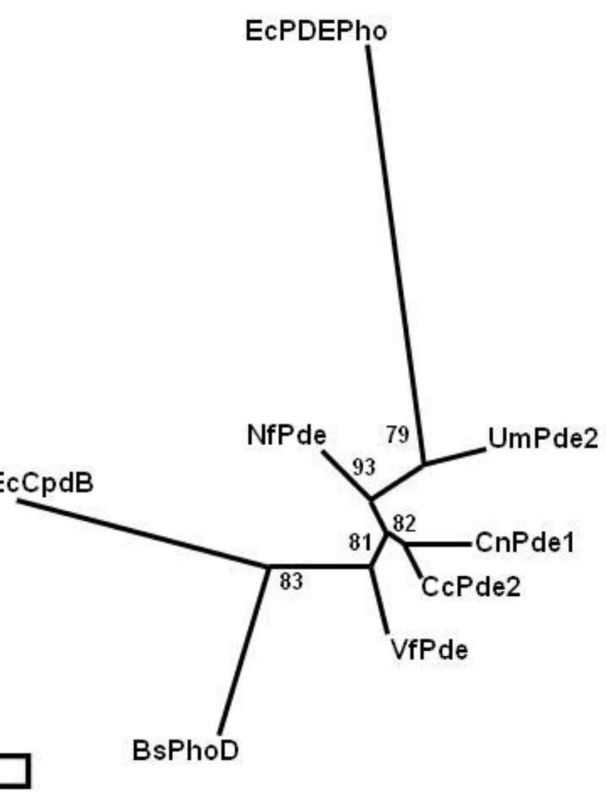

ECPDEPho UmPde2

ECPDEPho UmPde2

EcPDEPho UmPde2

ECPDEPhO UmPde2

ECPDEPho UmPde2

ECPDEPho UmPde2

ECPDEPho UmPde 2

ECPDEPho UmPde2

EcPDEPho UmPde2

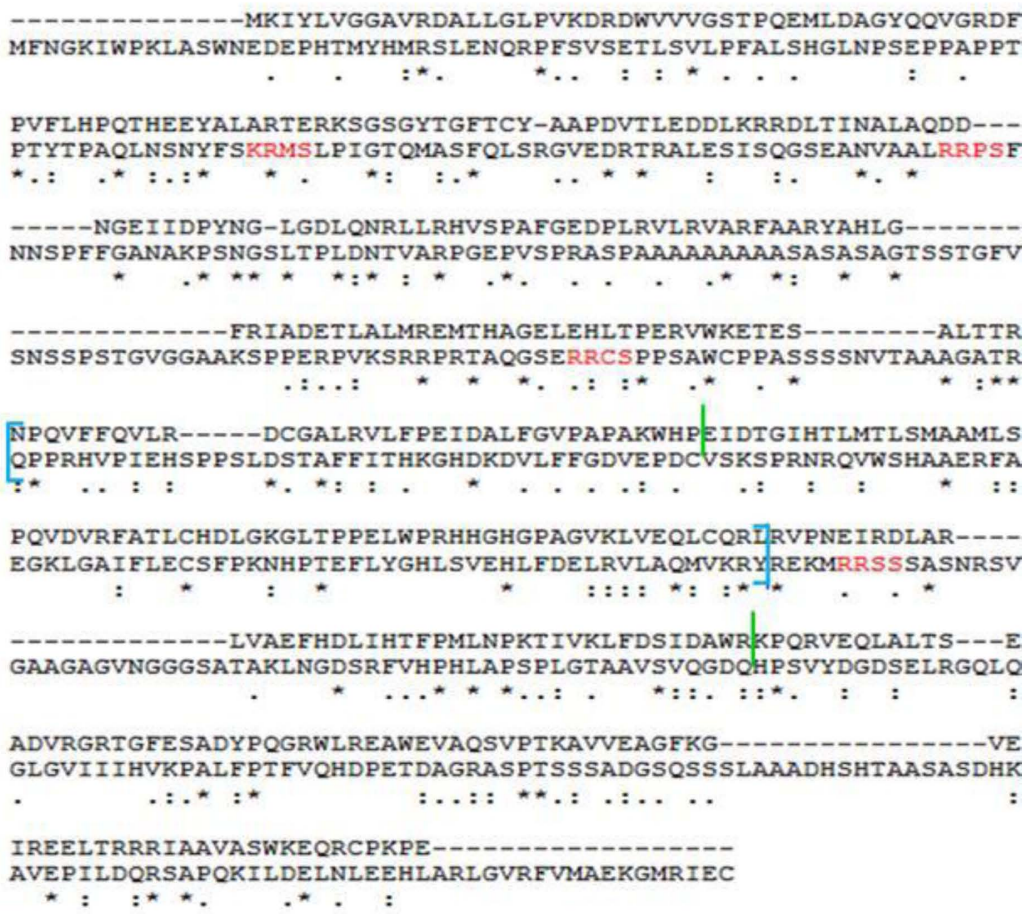

FIGURE 2 |Analysis of primary structure and phylogeny for UmPde1 and UmPde2. (A) Schematic diagram of UmPde1 with several closely related PDEs showing catalytic domain (indicated as large rectangles; COG 3379 for UmPde1, pfam 01663 for the remaining proteins) and transmembrane domain (small rectangles at $\mathrm{N}$-terminal portions of the respective proteins, along with amino acid residue numbers comprising the domains). " $\mathrm{P}$ " indicates putative phosphorylation site by PKA in UmPde1; * ${ }^{*}$, represents location of the $5^{\prime}$ primer used to produce a gene encoding a version of UmPde1 lacking the predicted membrane-spanning domain; (B) Alignment of UmPde2 primary sequence with that for EcPDEPho (NCBI Accession No. AP_003606). EcPDEPho is an Escherichia coli fused tRNA nucleotidyl transferase and 2'3'-cyclic phosphodiesterase/2'nucleotidase/phosphatase. The putative pfam02112, cAMP phosphodiesterase II domain, of UmPde2 is delineated by blue square brackets, while the HDc domain of EcPDEPho is bounded by green vertical lines. The predicted PKA target sites in UmPde2 are highlighted in red. (C) Unrooted tree showing relationship of UmPde2 catalytic domain compared to those of two PDEs from basidiomycetous and one ascomycetous fungi, as well as those from several bacteria. The EcCpdB (Accession No. P08331) and BsPhoD (Accession No. AAB47803) represent enzymes shown to have both cyclic phosphodiesterase and nucleotide phosphatase or cyclic phosphodiesterase and alkaline phosphatase activities, respectively. 
pyrophosphatase), um10895 (IPR000396, cAMP phosphodiesterase, class II), um04961 (IPR002073, 3' ,5'-cyclic nucleotide phosphodiesterase), um12014/um00004 (IPR004129, glycerophosphoryl ester phosphodiesterase), um00989 (IPR010347, tyrosyl-DNA phosphodiesterase), and um12190/um02441 (IPR0012386, 2' $3^{\prime}$-cyclic phosphodiesterase, plant; IPR009097, RNA ligase/cyclic nucleotide phosphodiesterase). With the exception of um02531 (annotated as "related to nucleotide diphosphatase"), all the remaining ORF entries were annotated as "conserved hypothetical proteins." We focused on ORFs um02531 and um10895, whose predicted InterPro domains and overall size matched more closely with participants in the cAMP-dependent PKA pathway governing cell morphology and pathogenicity of U. maydis. These ORFs, um02531 and um10895, will hereafter be referred to, respectively, as umpde1 and umpde2. A Conserved Domain Search at NCBI predicted that U. maydis UmPde1 contains a COG3379 domain, similar to pfam01663, type I phosphodiesterase/nucleotide pyrophosphatase, and UmPde2 contains a phosphodiesterase domain, pfam02112, for cAMP phosphodiesterase, class_II (See Figures 2A,B). The predicted UmPde1 also has a hydrophobic $\mathrm{N}$-terminal portion, suggesting that it contains a transmembrane region from amino acid 67 to amino acid 89. As seen in Figure 2A, this feature is also found in other fungal PDEs, and in human HsPdel and HsPde3A, B (not shown). Both UmPde1 and UmPde2 sequences were analyzed using TargetP for presence of signal peptides to evaluate any possible targeting to subcellular organelles. No such signal sequence was identified in either umpde1 or umpde2. Neither UmPde1 nor UmPde2 were predicted to contain any additional $\mathrm{N}$-terminal domains found in other PDE classes; GAF (cGMP-binding phosphodiesterase, Anabaena adenylyl cyclase, E. coli FhlA) domains common to a number of vertebrate classes of PDEs and to TcrPDEB1 and TcrPDEB2 from T. cruzi, were absent in UmPde1 and UmPde2.

Phosphodiesterases are a key component of the cAMP-PKA signaling pathway. Regulation of PKA pathway components is thus very important for virulence and growth of the fungus. Many components of the pathway are regulated by post-translational covalent modification (phosphorylation/dephosphorylation). To examine the potential for regulation by phosphorylation, both PDEs were assessed for PKA phosphorylation target sequences using NPS PROSCAN (Combet et al., 2000). UmPde1 was found to contain one putative PKA phosphorylation site (residues 64-67) while UmPde2 contains four putative PKA phosphorylation sites (Figure 2B). In addition, eight putative sites for phosphorylation by PKC were also predicted (not shown).

\section{EXPRESSION OF PDES}

Production of recombinant umpde1 and umpde2 ORFs cloned into the pET30b (+) vector failed in BL21 (DE3) pLysS (data not shown). To address potential problems with codon bias, expression was also attempted in Rosetta2 (DE3). UmPde2 was detectable as $\mathrm{a} \sim 56 \mathrm{kDa}$ band in the Rosetta2 (DE3) E. coli strain. Full-length UmPde1 was also not expressed in the Rosetta2 (DE3) strain when a predicted membrane-spanning domain was included. However, expression was detected in the Rosetta2 (DE3) when a derivative of the umpde1 gene, present in AA87UmPde1-pET30b, was used in which the region encoding the predicted membrane-spanning domain was deleted. Time course analysis of protein expression indicated that $4 \mathrm{~h}$ post-IPTG induction, protein expression did not increase significantly relative to total proteins, therefore the 4-h time point was used in all activity experiments.

\section{PHOSPHODIESTERASE ACTIVITY}

Phosphodiesterase activity was analyzed using freshly prepared crude protein extracts of $4 \mathrm{~h}$ induced cultures since no activity was detected in frozen samples. HPLC analysis was used to identify assay products and unreacted substrate relative to retention times of authentic standards. Background (empty vector) activity was subtracted from all samples to eliminate any potential endogenous E. coli activities.

When supplied with cAMP, both UmPde1 and UmPde2 produced a strong AMP product signal (Figure 3). In the vector alone sample (Figure 3A) one major cAMP peak was present and two minor peaks that correspond to AMP and adenosine were observed. UmPde1 showed a dominant AMP peak and minor cAMP and adenosine peaks (Figure 3B). In UmPde2 samples two major peaks that corresponded to AMP and adenosine and one minor cAMP peak were observed (Figure 3C). Specific activities for one set of replicates (shown in Figure 4) were calculated to be $8.62 \mathrm{nmol}$ AMP produced/min/mg protein and $1.10 \mathrm{nmol}$ adenosine produced $/ \mathrm{min} / \mathrm{mg}$ of protein for UmPdel and $6.61 \mathrm{nmol}$ AMP produced $/ \mathrm{min} / \mathrm{mg}$ protein and $4.76 \mathrm{nmol}$ adenosine produced $/ \mathrm{min} /$ mg protein for UmPde2. Thus, UmPdel is predominantly a cyclic PDE, while UmPde2 displays both PDE and phosphatase activities. Due to this latter finding, we conducted additional alignments of UmPde2 predicted amino acid sequence with those of two E. coli enzymes exhibiting dual functions ( $E$. coli PDEPho, Figure 2B, and E. coli $\mathrm{CpdB}$, not shown). There was a high degree of amino acid similarity with each and phylogenetic analyses placed UmPde2 together with EcPDEPho when compared with several other bacterial phosphodiesterases and with PDEs from basidiomycetous fungi (Figure 2C).

\section{SUBSTRATE SPECIFICITY PROFILE}

Based on computational analysis of the gene sequence, we predicted both UmPde1 and UmPde2 would contain cAMP phosphodiesterase activity. The discovery of a strong peak corresponding to adenosine in UmPde2 samples suggested the potential for an additional activity and necessitated deriving a more complete substrate specificity profile of both UmPde1 and UmPde2. The two most common cyclic nucleotides (cAMP and cGMP) with roles in signaling were analyzed as substrates for each enzyme. We found that UmPde1 was specific for cAMP and did not hydrolyze cGMP in these experiments (Table 1). In contrast, for UmPde2, along with conversion of cAMP into AMP, this enzyme was also able to convert cGMP into GMP (Table 1). In comparing complete metabolism of cyclic nucleotides into nucleoside $5^{\prime}$-monophosphates and nucleosides, UmPde2 displayed roughly threefold higher activity when supplied with cAMP compared to cGMP. In contrast, when supplied with nucleoside $5^{\prime}$-monophosphates, the rate of production of nucleosides was 1.8-fold higher for GMP compared with AMP.

UmPde1 did not convert nucleoside 5-monophosphates (AMP and GMP) into adenosine or guanosine, respectively (Table 1). UmPde1 and UmPde2 were also assayed with ADP and ATP as substrates. However, in the vector alone samples, ADP and ATP 


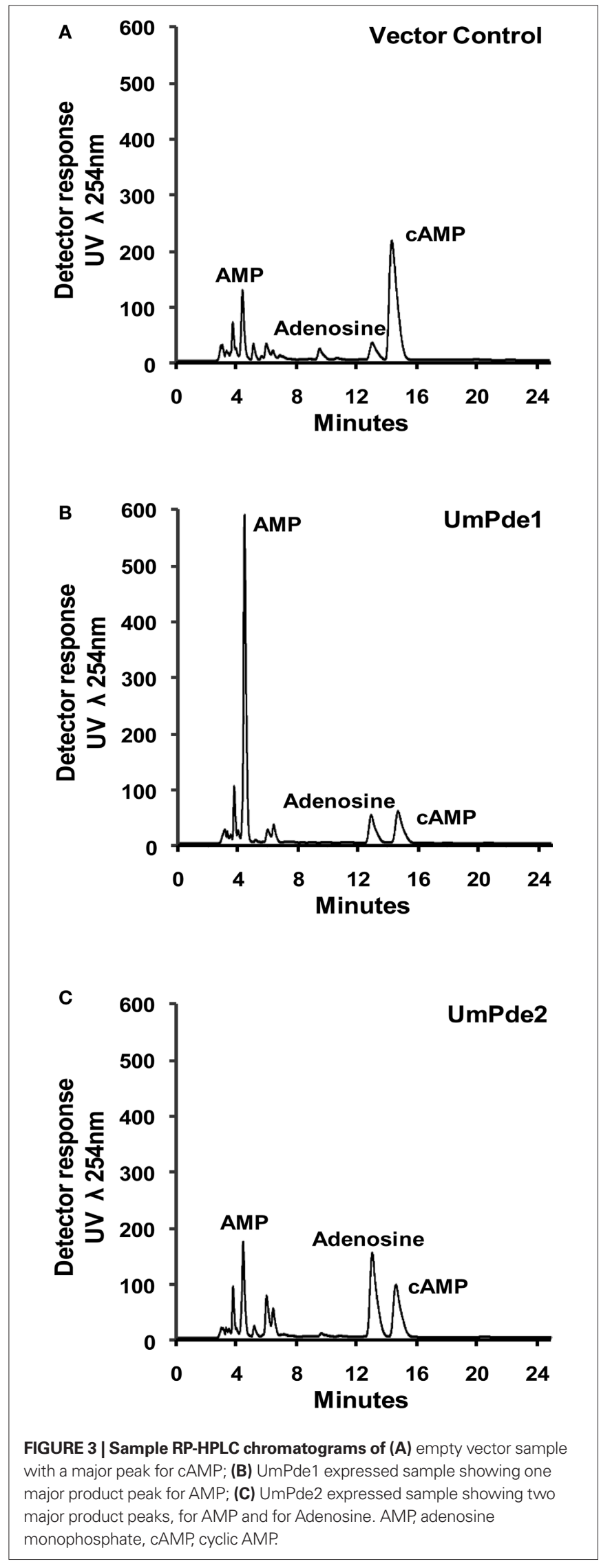

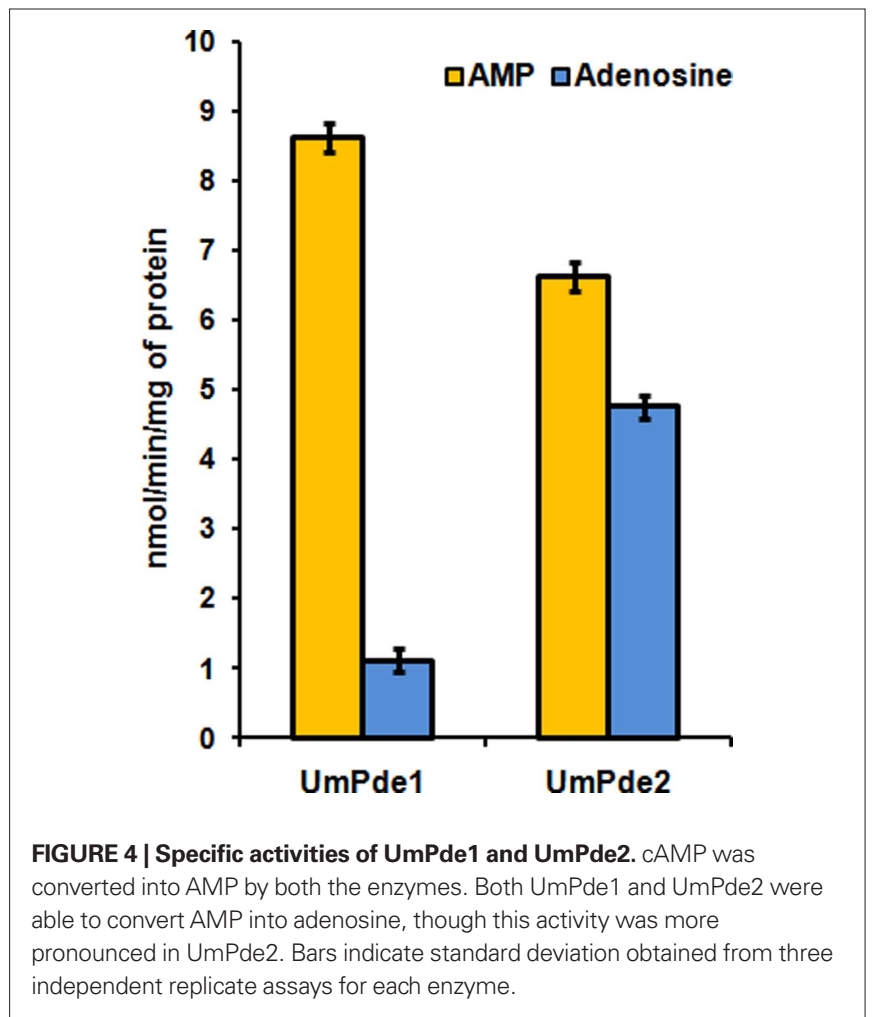

Table 1 | Substrate specificity for UmPde1 and Umpde2.

\begin{tabular}{lll}
\hline & \multicolumn{2}{c}{ \% Product } \\
\cline { 2 - 3 } Substrate used & UmPde1 & UmPde2 \\
\hline CAMP & 67 (AMP+Adeno) & 72 (AMP+Adeno) \\
CGMP & N.D. & 11 (Guano) \\
AMP & N.D. & 34 (Adeno) \\
ADP & N.D. & 34 (Adeno) \\
ATP & N.D. & 34 (Adeno) \\
GMP & N.D. & 63 (Guano)
\end{tabular}

Different nucleotides were used as substrates for UmPde1 and UmPde2 and their conversion into subsequent product was measured via RP-HPLC and tabulated for comparison. Activity in vector alone sample was subtracted as background activity.

N.D., none detected; Adeno, adenosine; Guano, guanosine; AMP, adenosine monophosphate.

were converted into AMP at a high rate and thus production of corresponding activity of UmPde1 and UmPde2 toward ATP, ADP or AMP could not be differentiated.

\section{RESPONSE TO IBMX}

To characterize UmPde1 and UmPde2 activity with respect to a known cyclic phosphodiesterase inhibitor and thus better define UmPde2 as a cyclic phosphodiesterase or nucleoside 5 '-monophosphatase, assays were conducted in the presence of IBMX. Three concentrations of inhibitor were tested $(10 \mu \mathrm{M}$, $500 \mu \mathrm{M}$, and $1 \mathrm{mM}$ ) and compared to vehicle control (DMSO only). 
Both UmPde1 and UmPde2 were found to be insensitive to IBMX at these concentrations, and yielded chromatograms comparable to the DMSO vehicle control (data not shown).

\section{DISCUSSION}

Important roles of cyclic nucleotides as second messengers in response to nutrient signals have been defined in many fungi (Rolland et al., 2002). As part of the overall system regulating cellular processes via second messengers, controlling internal levels of cyclic nucleotides is critical. While adenylate cyclases are responsible for synthesis of cyclic nucleotides, the flip side of the process are the PDEs that facilitate turnover of these molecules. In S. cerevisiae, two phosphodiesterases, Pde1 and Pde2, have been characterized and found to be low and high affinity PDEs, respectively. They are involved in modulating cAMP levels under different nutrient conditions, such as glucose availability, as addition of glucose to starved yeast cells lead to momentary increase in cAMP levels. Increased transcription of the gene for the low affinity phosphodiesterase, Pde1, is induced by addition of glucose via PKA and thus helps in the regulation of increased cAMP levels (Wera et al., 1997). In addition, there is evidence that phosphorylation of Pde1 and Pde2 plays a role in regulating their functions (Ma et al., 1999). Similarly, in $C$. neoformans, virulence factor production is regulated by the cAMP-PKA cascade and both Pde1 and Pde2, which are negatively regulated by $\mathrm{Pka} 1$ and $\mathrm{Pka} 2$ (catalytic subunits of PKA), are specifically required during melanin and capsule production under stress, e.g., low iron conditions. As with other PDEs examined in dimorphic fungi, UmPde1 and UmPde2 appear, from in silico analyses (Figure 2B), to also contain potential targets for regulation by protein kinases in U. maydis. Additional analyses of their predicted catalytic domains, suggested that each of these enzymes was phylogenetically similar to other PDEs containing the same pfam domains (Figure 5). In some cases, the clades identified in the analyses, for the most part reflected known substrate affinity profiles for the respective enzymes. For instance, most of the enzymes in the pfam00233/ HDc group (Figure 5) appeared to have a preference for cAMP. For the group II clade, both dual cAMP/cGMP specificity and IBMX resistance were found. On the other hand, substrate preference was not conserved in the group I clade. However, in both clades, insufficient biochemical data on substrate profiles and IBMX sensitivity prevent meaningful conclusions about these features in relation to the phylogenetic groupings. Regarding IBMX sensitivity, it is clear from examination of the pfam00233/HDac clade and numerous studies of human PDE families (Huai et al., 2004, Beavo et al., 2007) that IBMX sensitivity and PDE family do not go hand-inhand. Thus, the fact that both UmPde1 and UmPde2 are IBMX resistant, does distinguish them from other fungal PDEs characterized at this time, but does not directly provide insight into phylogenetic relationships among these and other PDEs. Additional insights about the $U$. maydis PDEs may be gained from comparisons made between the complete primary sequences of these proteins and their biochemical properties with those of other PDEs. Upon further biochemical analysis of these enzymes we confirmed that UmPde1 is specific for cAMP and does not hydrolyze cGMP in contrast to UmPde2, which was able to act, in its role as a PDE, on both cAMP and cGMP, with apparent preference for the former. UmPde2 also acts as a nucleotide phosphatase, thus showing dual functions both as a phosphodiesterase and as a phosphatase. Interestingly, here UmPde2 prefers GMP over AMP as a substrate. In yeast, Pde1 can convert both cAMP and cGMP into AMP and GMP, respectively, but the high affinity phosphodiesterase Pde2 was found to be involved in hydrolysis of cAMP specifically (Londesborough, 1977). Thus, UmPde2 shows biochemical similarity to Pde1 of S. cerevisiae and with the Pde1 of $D$. discoideum, which is also resistant to IBMX. UmPde2 also acts as a nucleotide phosphatase, thus showing dual functions both as a phosphodiesterase and as a phosphatase. In several bacteria it has been shown that phosphodiesterases can have dual function, acting both as phosphodiesterase as well as an AMP phosphatase (Liu et al., 1986; Fouts et al., 2008) or alkaline phosphatase (O’Brien and Herschlag, 2001; Zhang et al., 2005). For example, the E. coli and B. subtilis alkaline phosphatases were shown to have phosphodiesterase activity on bis- $p$-nitrophenyl phosphate, bis-pNPP (O'Brien and Herschlag, 2001). Interestingly, alignments of UmPde2 with two $E$. coli enzymes exhibiting dual functions ( $E$. coli $\mathrm{PDEPho}$, Figure 2B, and E. coli $\mathrm{CpdB}$, not shown) revealed a high degree of amino acid similarity and phylogenetic analyses placed UmPde2 together with EcPDEPho when compared with several other bacterial phosphodiesterases and with PDEs from basidiomycetous fungi (Figure 2C).

In comparing structural similarities of $U$. maydis UmPde1 with other fungal PDEs (and with several non-fungal PDEs; Figure 2A) we noticed that, like these enzymes, UmPdel has a hydrophobic $\mathrm{N}$-terminal region predicted to serve as a membrane-spanning domain and we expect it to be located in the plasma membrane of this organism. One possible significance of such a domain is the observation, discussed in several reports (Cooper, 2005; Scott, 2006; Smith and Scott, 2006; Alonso et al., 2007), of compartmentalization of cAMP and the importance in controlling the sub-cellular localization of enzymes involved in controlling available pools of this critical second messenger. This spatial organization of several pathway components is shown to be governed by A-kinase anchoring proteins (AKAPs). Elevations in the level of cAMP and PKA activity by different factors command different physiological outputs. This compartmentalization of cAMP-PDEs is shown to be important for tailoring individual cAMP responses and is because of spatially limited access to effectors of cAMP-PKA signaling pathway and PKA enzyme (Smith and Scott, 2006). While our prediction that UmPde1 spans the cytoplasmic membrane or is associated with it stems primarily from in silico predictions, further support for this assertion stems from our inability to express the full-length protein in E. coli with that domain present; it was successfully expressed as a truncated version removing that region. Consistent with the above fact, the derivative of UmPde1, with the deleted membrane-spanning domain, was not able to fully complement the multiple budding phenotype of UmPde1 deletion strains (unpublished observations). This suggests that UmPde1 might normally be located in the membrane of this organism. While this domain is not required for phosphodiesterase activity in vitro, it remains to be determined whether and what role such membrane localization might play in vivo.

In summary, at least six enzymes were predicted to have some sort of phosphodiesterase activity after inspection of the $U$. maydis genome and in this report; we have confirmed that UmPde1 and UmPde2 are phosphodiesterases, likely involved in the modulation of cAMP levels in U. maydis. Based on the putative phosphorylation 


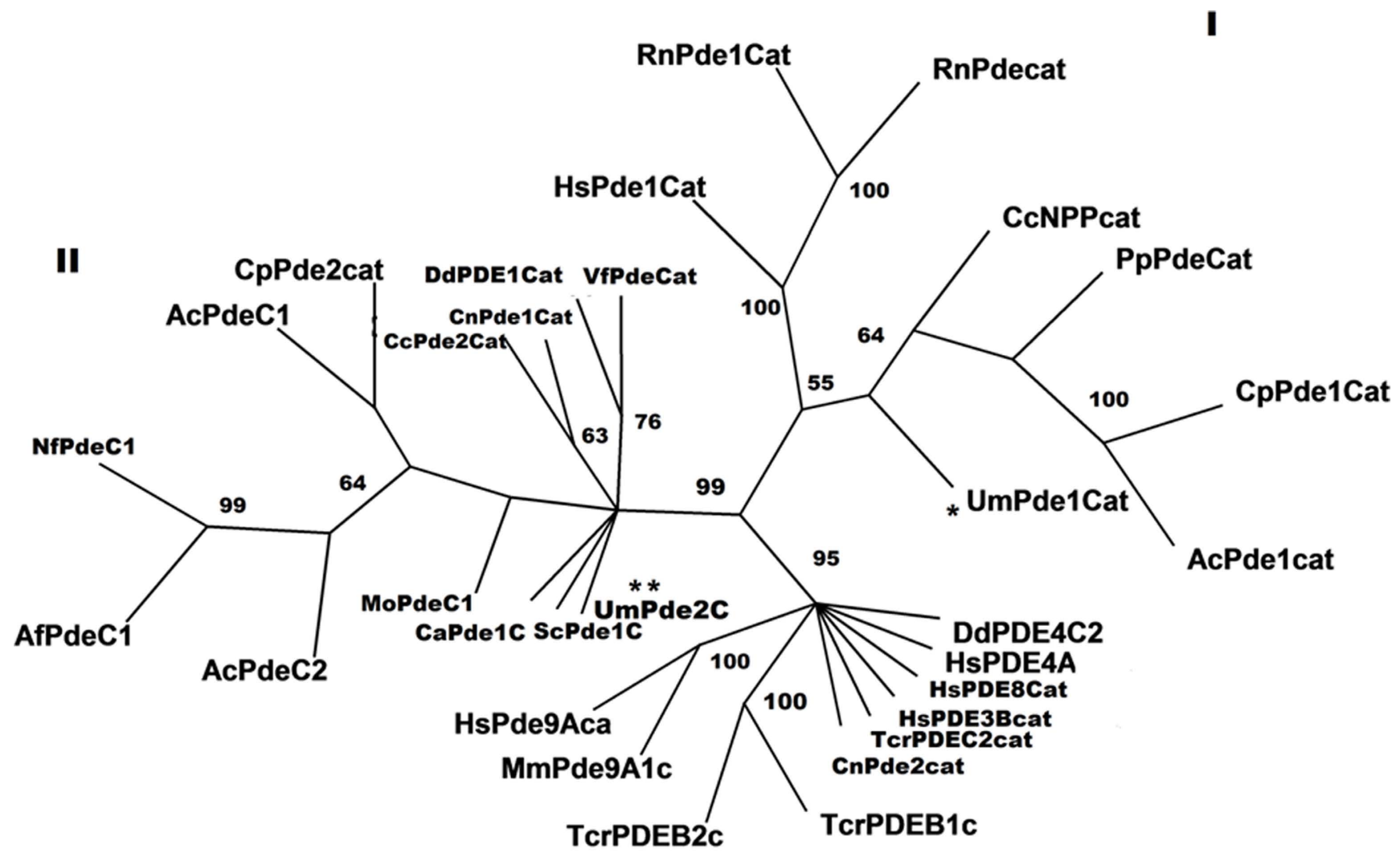

FIGURE 5 | Phylogenetic analyses for a variety of PDEs. This is an unrooted Phyl-ML tree from analysis of the catalytic domains of PDEs by Phylip as part of the seaview package of phylogenetic software (Gouy et al., 2010), employing theWAG model for ML analysis. Support for major branches is indicated from a tree showing the same topology, but generated by phylogenetic analysis in PAUP4.0b10 using a Full Heuristic search with Bootstrap analysis of 1000 replicates. The species abbreviations, NCBI or MUMDB (for the U. maydis PDEs) accession numbers, residues used, and taxonomy are: UmPde1Cat, Ustilago maydis, um02531, 120-465, Fungi, Basidiomycota; UmPde2Cat, U. maydis, um10895, 240-345, Fungi, Basidiomycota; CcNPPcat, Coprinopsis cinerea okayama7\#130, XP_001835792,105435, Fungi, Basidiomycota; PpPdeCat, Pichia pastoris GS115, XP_002490053, 140-180, Fungi, Ascomycota; CpPde1Cat, Coccidioides posadasii C735 delta SOWgp, XP_003070903, 223-553, Fungi, Ascomycota; HsPde1, Homo sapiens, NP_006199, 200-540, Mammalia, Primates; RnPde1Cat, Rattus norvegicus, BAA06333, 155-480, Mammalia, Rodentia; RnPdeCat, R. norvegicus, AAB61535, 160-480, Mammalia, Rodentia;TcrPDEB1c, Trypanosoma cruzi, AAM28249,674900, Euglenozoa, Kinetoplastida;TcrPDEB2c, T. cruzi, AAP49573,675-915, Euglenozoa, Kinetoplastida;TcrPDEC2cat, T. cruzi, AAY25022,673-600, Euglenozoa, Kinetoplastida; DdPDE1Cat, Dictyostelium discoideum AX4, XP_637948,98-448, Amoebozoa, Mycetozoa; DdPDE4C2, D. discoideum AX4, XP_636380,575-660, Amoebozoa, Mycetozoa; HsPDE3Bcat, H. sapiens, CAA64774, 800-960, Mammalia, Primates; CnPde1Cat, Cryptococcus neoformans var. neoformans

\section{pfam00233/HDc}

(Filobasidiella neoformans strain JEC21), XP_568076,45-455, Fungi, Basidiomycota; CnPde2cat, C. neoformans var. grubii (Filobasidiella neoformans serotype A), AAX73257,225-525, Fungi, Basidiomycota; ScPde1C, Saccharomyces cerevisiae S288c, NP_011266,4-368, Fungi, Ascomycota; CcPde2Cat, C. cinerea okayama7\#130, XP_001833477,45-400, Fungi, Basidiomycota; VfPdeCat, Vibrio fischeri, Q56686,25-322, Bacteria, Proteobacteria; CaPde1C, Candida albicans, P32782,32-420, Fungi, Ascomycota; MoPdeC1, Magnaporthe oryzae, XP_367803,18-180, Fungi, Ascomycota; AfPdeC1, Aspergillus fumigatus Af293, XP_752858,5-345, Fungi, Ascomycota; NfPdeC1, Neosartorya fischeri NRRL 181, XP_001264269,8-346, Fungi, Ascomycota; AcPdeC1, Aspergillus clavatus NRRL 1, XP_001268766,5-165, Fungi, Ascomycota; AcPdeC2, A. clavatus NRRL 1, XP_001268766,257-345, Fungi, Ascomycota; CpPde2Cat, C. posadasii C735 delta SOWgp, XP_003068881, 25-363, Fungi, Ascomycota; AcPde1Cat, A. clavatus NRRL 1, XP_001275664,210-545, Fungi, Ascomycota; MmPde9A1c, Mus musculus, EDL40358,252-480, Mammalia, Rodentia; HsPde9ACa, H. sapiens, NP_002597,252470, Mammalia, Primates; HsPde4A, H. sapiens, NP_001104777,195-435, Mammalia, Primates; HsPde8ACat, H. sapiens, EAX01966,550-810, Mammalia, Primates. I, clade including primarily PDEs containing a pfam01663 domain; II, clade consisting of pfam02112 domains from selected PDEs; pfam0233/HDc, clade containing the $3^{\prime}, 5^{\prime}$ cyclic nucleotide phosphodiesterase and/or metal-dependent phosphohydrolase (HDc) domain. Locations of UmPde1 and UmPde2 in the tree are emphasized with * and **, respectively. sites for PKA in both of these enzymes, further investigation of the regulation of these enzymes by phosphorylation via PKA is warranted. Moreover, the similarities of these fungal enzymes in several important respects to phosphodiesterases of other fungi, protozoans, and humans provides opportunities for interesting speculation about the evolution of these ubiquitous but diverse proteins.

\section{ACKNOWLEDGMENTS}

The authors would like to thank Dr. Muriel Mauer and Madhavi Jadhav at the University of Louisville for the use of her HPLC facility for inhibitor analysis. This work was supported in part, by an IRIG-RIG from the office of the Vice President for Research at the University of Louisville. 


\section{REFERENCES}

Abbott, B. M., and Thompson, P. E. (2005). A reversed-phase HPLC-based method for the assay of cyclic nucleotide phosphodiesterase activity. Anal. Biochem. 339, 185-187.

Alonso, G. D., Schoijet, A. C., Torres, H. N., and Flawia, M. M. (2006). TcPDE4, a novel membrane-associated cAMPspecific phosphodiesterase from Trypanosoma cruzi. Mol. Biochem. Parasitol. 145, 40-49.

Alonso, G. D., Schoijet, A. C., Torres, H. N., and Flawia, M. M. (2007). TcrPDEA1, a cAMP-specific phosphodiesterase with atypical pharmacological properties from Trypanosoma cruzi. Mol. Biochem. Parasitol. 152, 72-79.

Banuett, F., and Herskowitz, I. (1996). Discrete developmental stages during teliospore formation in the corn smut fungus, Ustilago maydis. Development 122, 2965-2976.

Barrett, K. J., Gold, S. E., and Kronstad, J. W. (1993). Identification and complementation of a mutation to constitutive filamentous growth in Ustilago maydis. Mol. Plant Microbe Interact. 6, 274-283.

Beavo, J. A., Houslay, M. D., and Francis, S.H., (2007). "Cyclic nucleotide phosphodiesterase superfamily," in Cyclic Nucleotide Phosphodiesterases in Health and Disease, eds J. A. Beavo, S. H. Francis, and M. D. Houslay (Boca Raton, FL: CRC Press), 3-17.

Bölker, M. (2001). Ustilago maydis - a valuable model system for the study of fungal dimorphism and virulence. Microbiology 147, 1395-1401.

Bölker, M., Urban, M., and Kahmann, R. (1992). The a mating type locus of $U$. maydis specifies cell signaling components. Cell 68, 441-450.

Combet, C., Blanchet, C., Geourjon, C., and Deleage, G. (2000). NPS@: network protein sequence analysis. Trends Biochem. Sci. 25, 147-150.

Cooper,D.M. (2005).Compartmentalization of adenylate cyclase and cAMP signalling. Biochem. Soc. Trans. 33 , 1319-1322.

D’Angelo, M. A., Sanguineti, S., Reece, J. M., Birnbaumer, L., Torres, H. N., and Flawia, M. M. (2004). Identification, characterization and subcellular localization of TcPDE1, a novel cAMP-specific phosphodiesterase from Trypanosoma cruzi. Biochem. J. 378, 63-72.

D'Souza, C. A., and Heitman, J. (2001). Conserved cAMP signaling cascades regulate fungal development and virulence. FEMS Microbiol. Rev. 25, 349-364.

Durrenberger, F., Wong, K., and Kronstad, J.W. (1998). Identification of a cAMPdependent protein kinase catalytic subunit required for virulence and morphogenesis in Ustilago maydis. Proc. Natl. Acad. Sci. U.S.A. 95, 5684-5689.

Emanuelsson, O., Nielsen, H., Brunak, S., and von Heijne, G. (2000). Predicting subcellular localization of proteins based on their N-terminal amino acid sequence. J. Mol. Biol. 300, 1005-1016.

Feldbrugge, M., Kämper, J., Steinberg, G., and Kahmann, R. (2004). Regulation of mating and pathogenic development in Ustilago maydis. Curr. Opin. Microbiol. 7, 666-672.

Fouts, D. E., Tyler, H. L., DeBoy, R. T., Daugherty, S., Ren, Q., Badger, J. H., Durkin, A.S., Huot, H., Shrivastava, S., Kothari, S., Dodson, R. J., Mohamoud, Y., Khouri, H., Roesch, L. F., Krogfelt, K. A., Struve, C., Triplett, E. W., and Methe, B.A. (2008). Complete genome sequence of the N2-fixing broad host range endophyte Klebsiella pneumoniae 342 and virulence predictions verified in mice. PLoS Genet. 4, e1000141. doi: 10.1371/journal.pgen.1000141.

Garcia-Pedrajas, M. D., and Gold, S. E. (2004).Kernelknowledge: smut of corn. Adv. Appl. Microbiol. 56, 263-290.

Garcia-Pedrajas, M. D., Nadal, M. Bölker, M., Gold, S. E., and Perlin, M. H. (2008). Sending mixed signals: redundancy vs. uniqueness of signaling components in the plant pathogen, Ustilago maydis. Fungal Genet. Biol. 45(Suppl. 1), S22-S30.

Gold, S., Duncan, G., Barrett, K., and Kronstad, J. (1994). cAMP regulates morphogenesis in the fungal pathogen Ustilago maydis. Genes Dev. 8, 2805-2816.

Gold, S. E., Brogdon, S. M., Mayorga, M. E., and Kronstad, J. W. (1997). The Ustilago maydis regulatory subunit of a cAMP-dependent protein kinase is required for gall formation in maize. Plant Cell 9, 1585-1594.

Gouy, M., Guindon, S., and Gascuel, O. (2010). SeaView version 4: a multiplatformgraphical userinterfacefor sequence alignment and phylogenetic tree building. Mol. Biol. Evol. 27, 221-224.

Hicks, J. K., Bahn, Y. S., and Heitman, J. (2005). Pde1 phosphodiesterase modulates cyclic AMP levels through a protein kinase A-mediated negative feedback loop in Cryptococcus neoformans. Eukaryotic Cell 4, 1971-1981.

Hoyer, L. L., Cieslinski, L. B., McLaughlin, M. M., Torphy, T. J., Shatzman, R., and Livi, G. P. (1994). A Candida albicans cyclic nucleotide phosphodiesterase: cloning and expression in Saccharomyces cerevisiae and biochemical characterization of the recombinant enzyme. Microbiology 140, 1533-1542.

Huai,Q.,Wang,H.,Zhang,W.,Colman, R.W., Robinson, H., and Ke, H. (2004). Crystal structure of phosphodiesterase 9 shows orientation variation of inhibitor 3 -iso- butyl-1-methylxanthine binding. Proc Natl. Acad. Sci. U.S.A. 101, 9624-9629.

Hunter, S., Apweiler, R., Attwood, T. K. Bairoch,A., Bateman,A., Binns, D., Bork, P., Das, U., Daugherty, L., Duquenne, L., Finn, R. D., Gough, J., Haft, D., Hulo, N. Kahn,D., Kelly,E.,Laugraud,A., Letunic I., Lonsdale, D., Lopez, R., Madera, M. Maslen, J., McAnulla, C., McDowall, J., Mistry,J.,Mitchell,A.,Mulder,N.,Natale, D., Orengo, C., Quinn, A. F., Selengut, J. D., Sigrist, C. J., Thimma, M., Thomas, P. D., Valentin, F., Wilson, D., Wu, C. H., and Yeats, C. (2009). InterPro: the integrative protein signature database. Nucleic Acids Res. 37, D211-D215.

Jung, W. H., Warn, P., Ragni, E., Popolo, L. Nunn, C. D., Turner, M. P., and Stateva, L. (2005). Deletion of PDE2, the gene encoding the high-affinity cAMP phosphodiesterase, results in changes of the cell wall and membrane in Candida albicans. Yeast 22, 285-294.

Kämper, J., Kahmann, R., Bölker, M., Ma, L. J., Brefort, T., Saville, B. J., Banuett, F., Kronstad, J. W., Gold, S. E., Muller, O., Perlin, M. H., Wosten, H. A., de Vries, R., Ruiz-Herrera, J., Reynaga-Pena, C. G., Snetselaar, K., McCann, M., PerezMartin, J., Feldbrugge, M., Basse, C. W. Steinberg, G., Ibeas, J. I., Holloman, W. Guzman, P., Farman, M., Stajich, J. E., Sentandreu, R., Gonzalez-Prieto, J. M. Kennell, J. C., Molina, L., Schirawski, J., Mendoza-Mendoza, A., Greilinger D., Munch, K., Rossel, N., Scherer, M. Vranes, M., Ladendorf, O., Vincon, V. Fuchs, U., Sandrock, B., Meng, S., Ho, E. C., Cahill, M. J., Boyce, K. J., Klose, J., Klosterman, S. J., Deelstra, H. J., OrtizCastellanos, L., Li, W., Sanchez-Alonso, P., Schreier, P. H., Hauser-Hahn, I. Vaupel, M., Koopmann, E., Friedrich, G., Voss, H., Schluter, T., Margolis, J., Platt, D., Swimmer, C., Gnirke, A. Chen, F., Vysotskaia, V., Mannhaupt, G., Guldener, U., Munsterkotter, M. Haase, D., Oesterheld, M., Mewes, H.W. Mauceli, E. W., DeCaprio, D., Wade, C. M., Butler,J., Young, S., Jaffe, D. B., Calvo, S., Nusbaum, C., Galagan, J., and Birren, B. W. (2006). Insights from the genome of the biotrophic fungal plant pathogen Ustilago maydis. Nature 444, 97-101.

Klose, J., de Sa, M. M., and Kronstad, J. W. (2004). Lipid-induced filamentous growth in Ustilago maydis. Mol. Microbiol. 52, 823-835.

Lee, N., D'Souza, C. A., and Kronstad, J.W. (2003). Of smuts, blasts, mildews, and blights: cAMP signaling in phytopathogenic fungi. Annu. Rev. Phytopathol. 41, 399-427.

Lengeler, K. B., Davidson, R. C., D'Souza, C., Harashima, T., Shen,W.C., Wang,P.,Pan, X., Waugh, M., and Heitman, J. (2000). Signal transduction cascades regulating fungal development and virulence. Microbiol. Mol. Biol. Rev. 64, 746-785.
Liu, J., Burns, D. M., and Beacham, I. R. (1986). Isolation and sequence analysis of the gene ( $\mathrm{cpdB}$ ) encoding periplasmic 2',3'-cyclic phosphodiesterase. J. Bacteriol. 165, 1002-1010.

Londesborough, J. (1977). Characterization of an adenosine $3^{\prime}: 5^{\prime}$-cyclic monophosphate phosphodiesterase from baker's yeast. Its binding to subcellular particles, catalytic properties and gel-filtration behaviour. Biochem. J. 163, 467-476.

Ma, P., Wera, S., Van Dijck, P., and Thevelein, J. M. (1999). The PDE1encoded low-affinity phosphodiesterase in the yeast Saccharomyces cerevisiae has a specific function in controlling agonist-induced cAMP signaling. Mol. Biol. Cell 10, 91-104.

Martinez-Espinoza, A. D., Ruiz-Herrera, J., Leon-Ramirez, C. G., and Gold, S. E. (2004). MAP kinase and cAMP signaling pathways modulate the $\mathrm{pH}$-induced yeast-to-mycelium dimorphic transition in the corn smut fungus Ustilago maydis. Curr. Microbiol. 49, 274-281.

Mehats, C., Andersen, C. B., Filopanti, M., Jin, S. L., and Conti, M. (2002). Cyclic nucleotide phosphodiesterases and their role in endocrine cell signaling. Trends Endocrinol. Metab. 13, 29-35.

Nikawa, J., Sass, P., and Wigler, M. (1987). Cloning and characterization of the low-affinity cyclic AMP phosphodiesterase gene of Saccharomyces cerevisiae. Mol. Cell. Biol. 7, 3629-3636.

O’Brien, P. J., and Herschlag, D. (2001). Functional interrelationships in the alkaline phosphatase superfamily: phosphodiesterase activity of Escherichia coli alkaline phosphatase. Biochemistry 40, 5691-5699.

Ramanujam, R., and Naqvi, N. I. (2010). PdeH, a high-affinity cAMP phosphodiesterase, is a key regulator of asexual and pathogenic differentiation in Magnaporthe oryzae. PLoS Pathog. 6, e1000897. doi: 10.1371/journal. ppat.1000897.

Regenfelder, E., Spellig, T., Hartmann, A., Lauenstein, S., Bölker, M., and Kahmann, R. (1997). G proteins in Ustilago maydis: transmission of multiple signals? EMBO J. 16, 1934-1942.

Rolland, F., Winderickx, J., and Thevelein, J.M. (2002). Glucose-sensing and -signalling mechanisms in yeast. FEMS Yeast Res. 2, 183-201.

Sass, P., Field, J., Nikawa, J., Toda, T., and Wigler, M. (1986). Cloning and characterization of the high-affinity cAMP phosphodiesterase of Saccharomyces cerevisiae. Proc. Natl. Acad. Sci. U.S.A. 83, 9303-9307.

Scott, J. D. (2006). Compartmentalized cAMP signalling: a personal perspective. Biochem. Soc. Trans. 34, 465-467. 
Smith, F. D., and Scott, J. D. (2006). Anchored cAMP signaling: onward and upward - a short history of compartmentalized cAMP signal transduction. Eur. J. Cell Biol. 85, 585-592.

Snetselaar, K. M., Bölker, M., and Kahmann, R. (1996). Ustilago maydis mating hyphae orient their growth toward pheromone sources. Fungal Genet. Biol. 20, 299-312.

Thompson, J. D., Higgins, D. G., and Gibson, T. J. (1994). CLUSTAL W: improving the sensitivity of progressive multiple sequence alignment through sequence weighting, position-specific gap penalties and weight matrix choice. Nucleic Acids Res. 22, 4673-4680.

Walter, R. D., and Opperdoes, F. R. (1982). Subcellular distribution of adenylate cyclase, cyclic-AMP phosphodiesterase, protein kinases and phosphoprotein phosphatase in Trypanosoma brucei. Mol. Biochem. Parasitol. 6, 287-295.

Wera, S., Ma, P., and Thevelein, J. M. (1997). Glucose exerts opposite effects on mRNA versus protein and activity levels of Pde1, the low-affinity cAMP phosphodiesterase from budding yeast, Saccharomyces cerevisiae. FEBS Lett. 420, 147-150.

Zhang, L., Balcerzak, M., Radisson, J., Thouverey, C., Pikula, S., Azzar, G., and
Buchet, R. (2005). Phosphodiesterase activity of alkaline phosphatase in ATP-initiated $\mathrm{Ca} 2+$ and phosphate deposition in isolated chicken matrix vesicles. J. Biol. Chem. 280, 37289-37296.

Conflict of Interest Statement: The authors declare that the research was conducted in the absence of any commercial or financial relationships that could be construed as a potential conflict of interest.

Received: 17 September 2010; accepted: 26 October 2010; published online: 29 November 2010.
Citation: Agarwal C, Schultz DJ and Perlin MH (2010) Two phosphodiesterases from Ustilago maydis share structural and biochemical properties with non-fungal phosphodiesterases. Front. Microbio. 1:127. doi: 10.3389/fmicb.2010.00127

This article was submitted to Frontiers in Fungi and Their Interactions, a specialty of Frontiers in Microbiology.

Copyright (C) 2010 Agarwal, Schultz and Perlin. This is an open-access article subject to an exclusive license agreement between the authors and the Frontiers Research Foundation, which permits unrestricted use, distribution, and reproduction in any medium, provided the original authors and source are credited. 